\title{
Alev Püskürtme Piroliz Yöntemi Kullanarak IGZO Nanopartiküllerinin Sentezi ve Karakterizasyonu
}

\author{
Serdar YILDIRIM ${ }^{1,2 *}$ \\ ${ }^{1}$ Metalurji ve Malzeme Mühendisliği Bölümü, Mühendislik Fakültesi, Dokuz Eylül Üniversitesi, İzmir, Türkiye \\ ${ }^{2}$ Nanobilim ve Nanomühendislik A.B.D., Fen Bilimleri Enstitüsü, Dokuz Eylül Üniversitesi, İzmir, Türkiye \\ (ORCID: 0000-0002-3730-3473)
}

\begin{abstract}
Öz
Yüksek elektron mobilitesi, düşük eşik gerilimi ve kaplama sonrası şeffaf özellik gösteren İndiyum Galyum Çinko Oksit (InGaZnO 4 , IGZO) malzemesi ekran teknolojilerinde artan bir ivmeyle kullanılmaktadır. Bu çalışmada, IGZO nanopartikülleri alev püskürtme piroliz yöntemi kullanılarak tek aşamada başarıyla sentezlendi. Üretilen nanopartiküllerin faz ve element analizleri, sırasıyla X-1şını kırınımı (XRD) ve X-1şını fotoelektron spektroskopisi (XPS) ölçümleriyle yapıldı. Isıl işlem öncesi XRD analizinde amorf yapı gözlemlenirken, $1200{ }^{\circ} \mathrm{C}$ de yapılan kalsinasyon sonucu rombohedral kristalin $\mathrm{InGaZnO}_{4}$ yapısı tespit edildi. Elementel analiz sonucunda yap1 içerisinde In, Ga, Zn ve O elementlerinin varlığı kanıtlandı. Yüzey morfolojisi ve partikül büyüklüğü taramalı elektron mikroskobu (SEM) kullanılarak tespit edilirken, nanopartiküllerin parçacık boyutlarının $<100 \mathrm{~nm}$ den küçük ve yarı küresel olduğu bulundu. UV uyarma altında nanopartiküllerin fotolüminesans ve optik bozulma zamanı karakterizasyonları incelenmiş ve $380 \mathrm{~nm}$ uyarma altında IGZO nanopartikülleri, $510 \mathrm{~nm}$ ve $570 \mathrm{~nm}$ civarında emisyon sergilemiştir. Bu uyarma ve emisyon altında yapılan optik bozulma süresi iki üstelli ve 32,36 $\mu$ s olarak hesaplanmıştır.
\end{abstract}

Anahtar kelimeler: IGZO, Alev püskürtme, Nanopartikül, Fotolüminesans.

\section{Synthesis and Characterization of IGZO Nanoparticles Using Flame Spray Pyrolysis Method}

\begin{abstract}
The Indium Gallium Zinc Oxide ( $\mathrm{InGaZnO}_{4}$, IGZO) material, which shows high electron mobility, low threshold voltage and transparent after coating, has been used with increasing acceleration in screen technologies. The flame spray pyrolysis technique was used to successfully synthesize IGZO nanoparticles in one step in this study. X-ray diffraction (XRD) and X-ray photoelectron spectroscopy (XPS) measurements were used to determine the phase and elemental composition of the nanoparticles, respectively. While the amorphous structure was observed in the $\mathrm{XRD}$ analysis prior to the heat treatment, the $\mathrm{InGaZnO}_{4}$ structure of the rhombohedral crystal was determined through calcination at $1200{ }^{\circ} \mathrm{C}$. As a result of elemental analysis, the presence of $\mathrm{In}, \mathrm{Ga}, \mathrm{Zn}$ and $\mathrm{O}$ elements in the structure was proven. The scanning electron microscopy (SEM) was used to determine the surface morphology and particle size of nanoparticles, which were found to be less than $100 \mathrm{~nm}$ in size and hemispherical. The photoluminescence and optical decay time characterizations of nanoparticles were studied under UV excitation and IGZO nanoparticles exhibited emission around $510 \mathrm{~nm}$ and $570 \mathrm{~nm}$ at $380 \mathrm{~nm}$ excitation. Under these conditions of excitation and emission, the optical decay time was computed as bi-exponentials and $32.36 \mu \mathrm{s}$.
\end{abstract}

Keywords: IGZO, Flame spray, Nanoparticle, Photoluminescence.

\footnotetext{
*Sorumlu yazar: serdar.yildirim@deu.edu.tr

Geliş Tarihi: 02.04.2021, Kabul Tarihi: 13.09.2021
} 


\section{Giriş}

İnce film transistörlerde (IFF'ler), örneğin çinko oksit ( $\mathrm{ZnO}$ ) [1], galyum oksit [2], kalay oksit [3], indiyum oksit [4], indiyum çinko oksit (IZO) [5], çinko kalay oksit (ZTO) [6] ve indiyum galyum çinko oksit (IGZO) [7, 8] gibi kanal tabakası olarak kullanılan metal oksit esaslı malzemeler, esnek elektronik cihazlar, optoelektronik ve mobil ekranlar gibi uygulamalar için geniş alanda çalışılmaktadır. Özellikle, IGZO, şeffaf TFT uygulamaları için çok umut verici bir malzeme olup büyük bant aralığ 1 ve geniş taşıyıcı konsantrasyonları kontrol edebilirlik sağlar. Ek olarak, IGZO üniform bir amorf faz olarak biriktirilebilir ve yine de yüksek elektriksel mobilite sergiler [10]. Bunun nedeni, IGZO' nun sadece kristal fazda değil, amorf fazda da yüksek performans göstermesidir. Amorf fazdaki yüksek performans, geçiş sonrası metal katyonların küresel s-orbitallerindeki minimum bir iletken bant oluşumundan kaynaklanmaktadır. Bu küresel s-orbitalleri, maksimum değerlik bandını oluşturan oksijen 2porbitallerinden önemli ölçüde daha büyüktür. Metal-oksijen-metal bağlarında bir bozulma meydana gelse bile, komşu metalik s-orbitaller arasındaki büyük doğrudan örtüşme taşıyıcı taşınmasını sağlar [11].

Özellikle, amorf IGZO (a-IGZO) TFT'ler, düşük sıcaklıklarda ve hatta oda sıcaklığında (RT) yüksek hareketlilik ve akım açma-kapama oranı $\left(\mathrm{I}_{\text {on }} / \mathrm{I}_{\text {off }}\right)$ nedeniyle a-Si TFT'lere veya organik TFT'lere göre daha çekici bir malzemedir. Bununla birlikte, a-IGZO filmlerin çoğu, darbeli lazer biriktirme, radyo frekansı magnetron püskürtme ve atomik tabaka biriktirme gibi vakum işlemleriyle üretilir [12-18]. Bu cihazlarda üretim yapılabilmesi için de kaplama yapılacak hedef malzemeye ihtiyaç duyulur. Bu hedef malzemeler de çeşitli nanopartikül üretim yöntemleriyle üretilerek şekillendirilip özel olarak kullanılmaktadır. Bu çalışmanın amacı da üretimi son derece zor ve karmaşık olan bu malzemenin farklı üretim tekniği kullanılarak nanopartikül olarak üretimini gerçekleştirip karakterize etmektir. IGZO gibi saf karışı metal oksit tozlarının sentezi oldukça zordur. Nanopartiküllerin sentezi için sol-gel [19], hidrotermal [20], modifiye yanma [21], katı hal seramik yöntemi, yanma tekniği, 1slak kimyasal sentez yöntemi ve tek aşamalı alev püskürtme piroliz (FSP) tekniği gibi uygulanan birçok farklı teknik vardır. Nanoparçacıkların sentezlenmesi için, FSP, sentez zamanını ve artan reaksiyon oranını düşürmesi nedeniyle en çok tercih edilen tekniklerden biridir. Tekniğin işlem üzerinde kontrol basitliği ve tekrar üretilebilirliği, başlangıç malzemesinin yüksek hızlı püskürtme jeti kullanarak alevin içine püskürtülmesinin kolaylığı, hazırlanan başlangıç malzemesinin doğrudan yakıtta dağılım potansiyeli ve daha hızlı aerosol oluşumu gibi birçok avantajı vardır. Bu nedenle, FSP, nano ölçekli metal oksitlerin ve karmaşık metal oksitlerin üretiminde birçok kez uygulanmıştır.

$\mathrm{Bu}$ çalışmada, daha önce yapılan literatür çalışmaları incelendiğinde ilk kez IGZO nanopartikülleri FSP yöntemi kullanılarak sentezlenmiştir. Ayrıca partiküllerin yapısal, kimyasal ve optik özellikleri karakterize edilmiştir.

\section{Materyal ve Metot}

IGZO nanopartiküllerinin alev sprey piroliz yöntemi ile üretiminde ön başlatıcı olarak İndiyum nitrat hidrat $\left(\mathrm{In}\left(\mathrm{NO}_{3}\right)_{3} \mathrm{xH}_{2} \mathrm{O}\right)$, Galyum nitrat hidrat $\left(\mathrm{Ga}\left(\mathrm{NO}_{3}\right)_{3} \times \mathrm{XH}_{2} \mathrm{O}\right)$ ve Çinko nitrat tetrahidrat $\left(\mathrm{Zn}\left(\mathrm{NO}_{3}\right) .4 \mathrm{H}_{2} \mathrm{O}\right)$; çözücü olarak 2-Metoksietanol $\left(\mathrm{C}_{3} \mathrm{H}_{8} \mathrm{O}_{2}\right)$ kullanılmıştır. Ön başlatıcılar SigmaAldrich'ten tedarik edilmiştir. Yüksek saflıkta kullanılan ürünler için ayrıca saflaştırma işlemi gerçekleştirilmemiştir.

\subsection{Solüsyonların Hazırlanması}

Başlangıç malzemesi olarak kullanılan Galyum Nitrat Hidrat, İndiyum Nitrat Hidrat ve Çinko Nitrat Tetrahidrat farklı beherlerde 2-Metoksietanol içerisine eklenmiş ve karışım 0,2 M olacak şekilde çözünmeye bırakılmıştır. Çözeltiler manyetik karıştırıcı ile homojen karışım sağlamak amacıyla karıştırılmıştır. Homojen karışım sağlandıktan sonra 3 çözelti bir beherde birleştirilmiş ve şeffaf bir çözelti elde edilmesi için $80{ }^{\circ} \mathrm{C}$ de 60 dakika karıştırılmıştır. Çözeltinin tam çözünüp çözünmediğini belirlemek için türbidimetre ile bulanıklığına bakılmıştır. Çözeltinin ortalama türbiditesi 1,25 ntu olarak bulunmuştur. Bu değer solüsyonun homojen bir şekilde çözündüğünü göstermektedir. Çözeltiler, Tablo 1 e göre hazırlanmıştır. 
Tablo 1. IGZO üretimi için kullanılan malzemeler ve miktarları

\begin{tabular}{llll}
\hline & Malzeme & Miktar & Mol \\
\cline { 2 - 4 } Ön & $\mathrm{In}\left(\mathrm{NO}_{3}\right)_{3} \cdot \mathrm{xH}_{2} \mathrm{O}$ & $7,522 \mathrm{~g}$ & $0,02 \mathrm{~mol}$ \\
başlatıcılar & $\mathrm{Ga}\left(\mathrm{NO}_{3}\right)_{3} \cdot \mathrm{xH}_{2} \mathrm{O}$ & $6,3943 \mathrm{~g}$ & $0,02 \mathrm{~mol}$ \\
& $\mathrm{Zn}\left(\mathrm{NO}_{3}\right) \cdot 4 \mathrm{H}_{2} \mathrm{O}$ & $1,307 \mathrm{~g}$ & $0,02 \mathrm{~mol}$ \\
Çözücü & $\mathrm{C}_{3} \mathrm{H}_{8} \mathrm{O}_{2}$ & $100 \mathrm{ml}$ & \\
\hline
\end{tabular}

\subsection{Nanopartiküllerin Sentezi}

Alev spreyi ile sentezlenen nanopartiküllerin üretimi önceki çalışmalara göre yapılmıştır [22, 23]. Np10'un (Tethis, Milan, İtalya) alevli sprey piroliz ekipmanı deneyler boyunca kullanıldı. Şekil 1, alev püskürtme piroliz ekipmanının deneysel kurulumunu göstermektedir. Hazırlanan IGZO çözeltisi, alev püskürtme piroliz ekipmanının çözelti şırıngasına beslendi. Alev püskürtme reaktöründe, sıvı çözelti 5 $\mathrm{mL} /$ dak bir besleme hızına sahip bir şırınga pompası ile bir Metan (1,5 L/dak) / oksijen (3,0 L / dak)yakıt/oksijen oranı ile oluşan püskürtme alevine gönderildi ve ince sprey oluşturmak için oksijenle dağıtıldı. FSP ile sentezlenen partiküller, bir vakum pompası tarafindan çekilen egzoz gazları ile bir cam mikrofiber filtreyi destekleyen su soğutmalı, paslanmaz çelik bir filtre muhafazası kullanılarak toplandı. Sentezlenen partiküllere yapıda kalan organiklerin uzaklaştırılması ve kristaliniteyi arttırmak için 1200 ${ }^{\circ} \mathrm{C}$ 'de 4 saat kalsinasyon işlemi yapıldı.

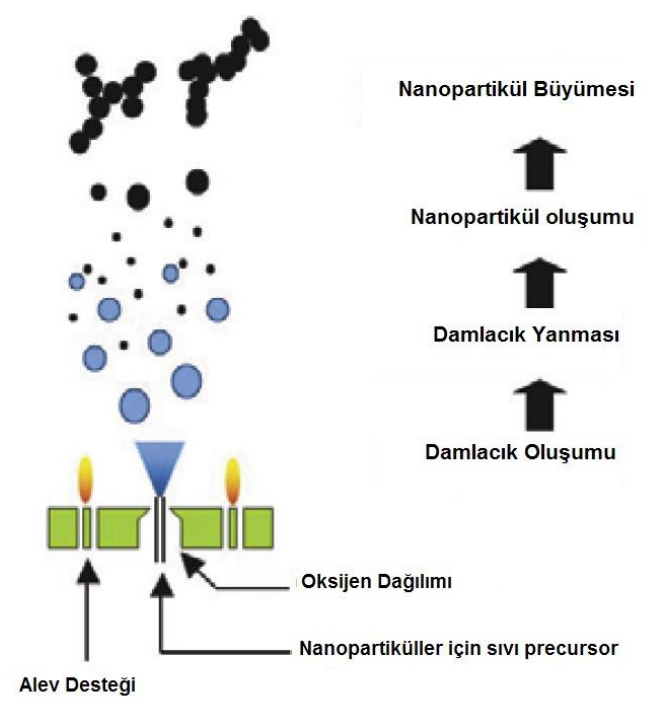

Şekil 1. Alev sprey piroliz sisteminin şematiği [24]

\subsection{Karakterizasyon}

Hazırlanan IGZO partiküllerinin faz ve kristal yapılarının belirlenmesi, $45 \mathrm{kV}$ voltaj ve $44 \mathrm{~mA}$ akım ayarları ile çalışan ve $\mathrm{Cu}-\mathrm{K}_{\alpha}$ radyasyonu $(1,5405 \AA)$ kullanan bir X-ışını difraktometresi (XRD, Thermo Scientific ARL) ile yapıldı. XRD verileri, $20^{\circ} \leq 2 \theta \leq 70^{\circ}$ aralığında $2 \%$ dak tarama hızında kaydedildi. Monokromatik bir Al-Ka (1486,7 eV) X-1şını kaynağ ve $400 \mathrm{~nm}$ çapında bir 1şın büyüklüğü ile X-1şını fotoelektron spektroskopisi (XPS, Thermo Scientific K-Alpha), toz numune için element bileşimini ve yüzey kimyasını belirlemek için gerçekleştirildi. Cihaz altın elementinin $4 \mathrm{f}_{7 / 2}$ bağlanma enerjisine göre kalibre edildi. Veri toplama süresi boyunca sistemin basınc1 $5 \times 10^{-10}$ mbar'1n altında tutulmuştur. Genel taramanın XPS verileri, $150 \mathrm{eV}$ geçiş enerjisi uygulayan $1 \mathrm{eV}$ tarama hızı ile -10 ile $1350 \mathrm{eV}$ bağlanma enerjileri arasında gerçekleştirildi. Isıl işlem öncesi ve sonrası nanopartiküllerin mikroyapı görüntüleri, alan etkili taramalı elektron mikroskobu (FESEM, Zeiss Carl Ultra Plus) kullanılarak 100 kX, 200 kX büyütmelerde ve $10 \mathrm{kV}$ hızlandırıcı voltajda gerçekleştirildi. Kararlı hal fotoluminesans (PL) emisyon spektrumları Edinburgh cihazının FLSP 920 floresan spektrometresi kullanılarak ölçülmüştür. Floresan bozulma süresi ölçümleri, FLSP 920'nin tek foton sayma tekniği (TCSPC) ile yapıldı. Cihazda sırasıyla kararlı hal ve bozunma zamanı ölçümleri için standart bir $15 \mathrm{~W}$ xenon lamba ve mikro saniye flaş lambası kullanıldı. 


\section{Bulgular ve Tartışma}

\subsection{Faz Analizi}

Gerekli FSP üretimi ve $1200{ }^{\circ} \mathrm{C}$ kalsinasyon sonrası elde edilen partiküllerin faz yapıları Şekil 2'de gösterilmiştir. FSP sonras1 yap1 incelendiğinde partiküllerin kristalin olmadığ ve amorf bir yap1 sergilediği görülmektedir. $1200{ }^{\circ} \mathrm{C}$ kalsinasyon sonrası yapıda tamamen rombohedral kristalin $\mathrm{InGaZnO}_{4}$ (IGZO, JCPDS:38-1104) fazı elde edildiği gözlemlenmiştir. Elde edilen pikler literatürle de benzer sonuçlar göstermiştir [10, 25].

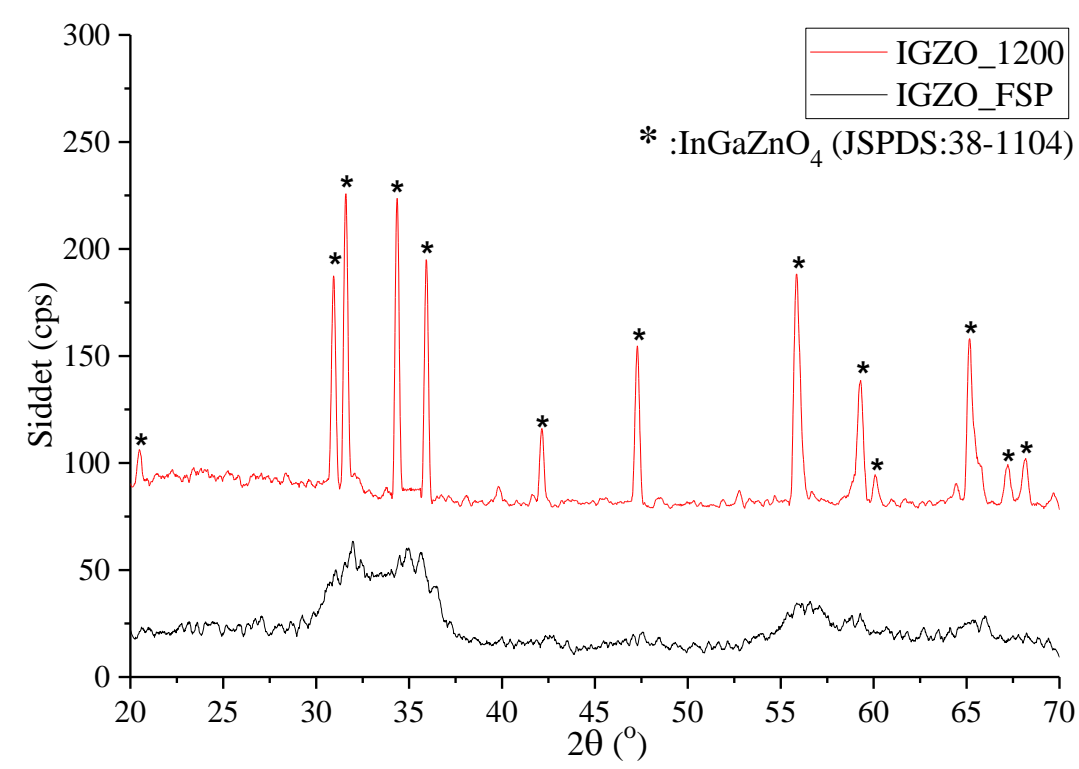

Şekil 2. FSP ve 1sıl işlem sonrası IGZO nanopartiküllerinin XRD desenleri

\subsection{Elementsel Analiz}

FSP yöntemiyle sentezlenen IGZO nanopartiküllerin stokiyometrik olarak doğru kompozisyonda üretilip üretilmediğini ve ortam-1sıl işlem kaynaklı kirlilik içerip içermediğini tespit etmek için X1şınları fotoelektron spektroskopi (XPS) cihazıyla elementel olarak analiz yapılmıştır. Şekil 3'te 1200 ${ }^{\circ} \mathrm{C}$ de kalsine edilmiş IGZO nanopartiküllerin XPS genel tarama grafiği gösterilmiştir. Sonuçlara baktığımızda In3d5, O1s, Zn2p3 ve Ga2p3 elementleri sirasiyla $445 \mathrm{eV}, 531 \mathrm{eV}, 1022,78 \mathrm{eV}$ ve 1119 $\mathrm{eV}$ bağlanma enerjilerinde tespit edilmiştir. Bu bağlanma enerjilerindeki metal elementlerinin oksit formunda olduğunu söyleyebiliriz [26-28].

Ayrıca ağırlıkça \% oranlarına baktığımızda $\mathrm{InGaZnO}_{4}$ kompozisyonunu doğrulamaktadır. Sonuçlar Tablo 2'de ayrıntılı bir şekilde verilmiştir. Tespit edilen elementler haricinde başka bir element gözlemlenmemiştir. Bu da malzemenin kirlilik içermediğini başarılı bir şekilde istenilen kompozisyonda üretildiğini göstermektedir.

Tablo 2. IGZO_1200 nanopartiküllerin XPS analiz sonuçları

\begin{tabular}{lcc}
\hline Element & Bağlanma Enerjisi (eV) & Ağırlıkça $(\%)$ \\
\hline Zn2p3 & 1022,78 & 17,83 \\
O1s & 531,46 & 23,73 \\
In3d5 & 445 & 37,09 \\
Ga2p3 & 1119,01 & 21,35 \\
\hline
\end{tabular}




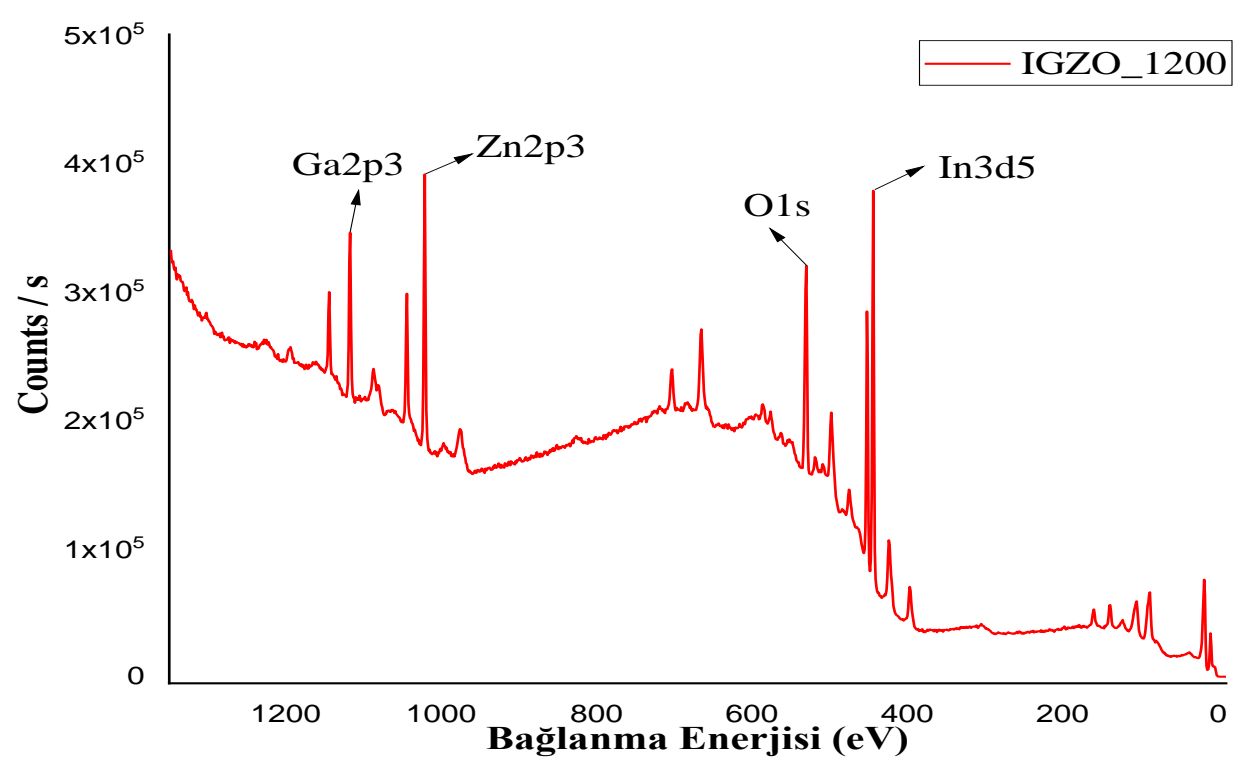

Şekil 3. IGZO_1200 nanopartiküllerin XPS genel tarama grafiği

\subsection{Partikül Morfolojisi}

FSP Isıl işlem öncesi ve sonrası partiküllerin morfolojileri Şekil 4'te gösterilmiştir. Şekil 4a-b, FSP sonrası alınan $100 \mathrm{kX}$ ve $200 \mathrm{kX}$ büyütmelerde alınan SEM görüntüleridir. Görüntüler incelendiğinde partiküllerin yarı küresel formda aglomere halinde ve ortalama 20-30 nm civarında olduğu gözlemlenmiştir. $1200^{\circ} \mathrm{C}$ de yapılan kalsinasyon sonrası tanelerin boyutları artmış ve $40-60 \mathrm{~nm}$ partikül boyutlarında olduğu Şekil 4c-d de gösterilmiştir. Taneler yarı-küresel ve küpe benzer şekilde olduğu görülmüştür. Şekil 4d deki tane şekilleri XRD sonucu tespit edilen rombohedral (kübik yapı) kristal yapısını desteklemektedir. FSP üretim yöntemiyle tek aşamada $100 \mathrm{~nm}$ ' nin altında partiküllerin sentezlendiği SEM görüntüleriyle ispatlanmıştır.
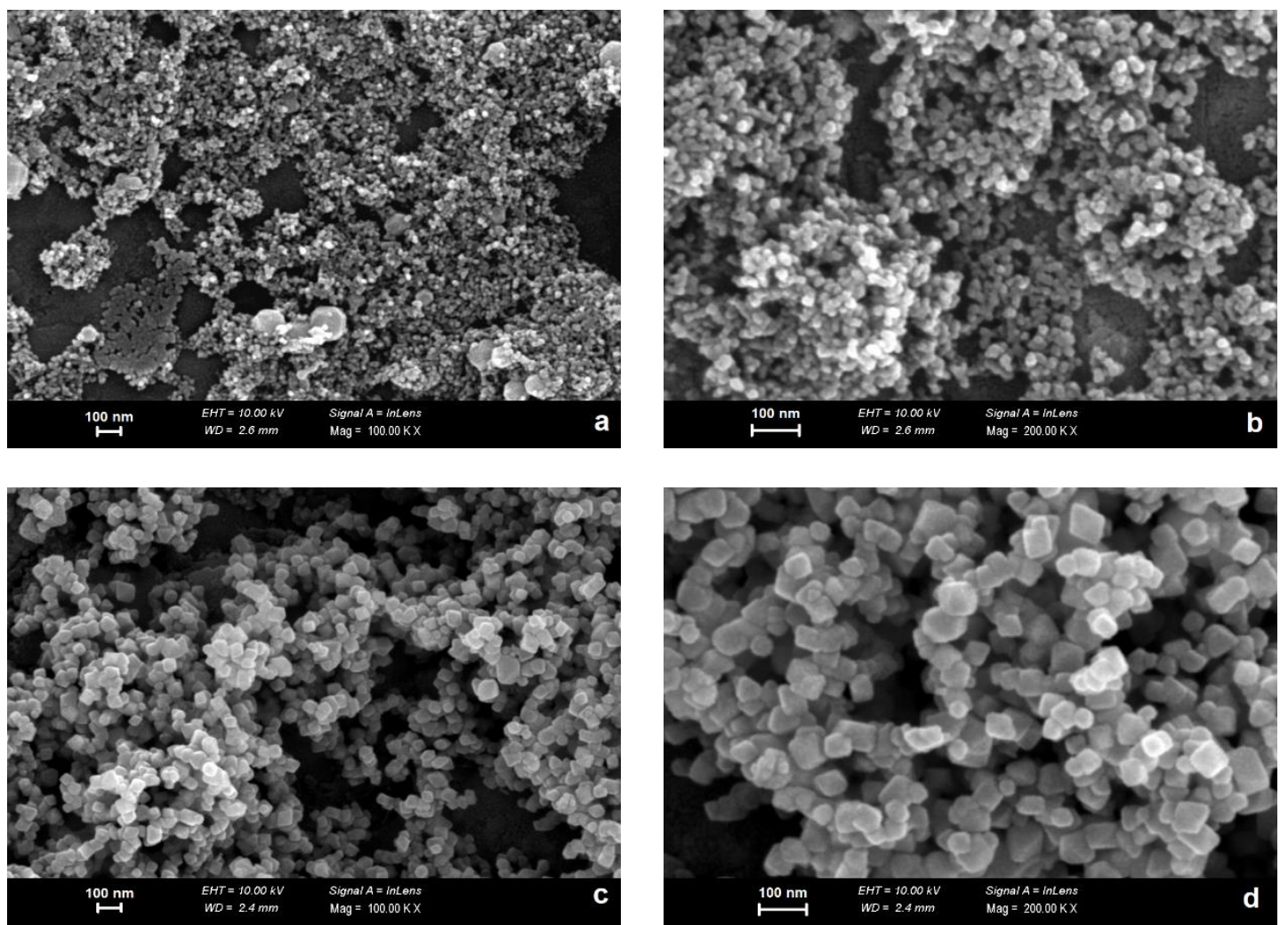

Şekil 4. IGZO Partiküllerin 1sıl işlem öncesi ve sonrası SEM görüntüleri a) IGZO_FSP (100 kX), b) IGZO_FSP $(200 \mathrm{kX})$, c) IGZO_1200 (100 kX), d) IGZO_1200 (200 kX) 


\subsection{Fotolüminesans Özellikler}

Şekil 5'te IGZO_1200 nanopartikülün uyarma ve emisyon band yapıları gösterilmiştir. Partiküller 380 $\mathrm{nm}$ uyarma altında 510 ve $570 \mathrm{~nm}$ dalga boyların 2 ayrı emisyon piki vermiştir. Bu pikler iletim bandındaki elektronlara veya iletken bandın yakınındaki sığ donör seviyelerine ve değerlik bandının maksimumu üzerinde tuzaklanmış alıcılar arasındaki rekombinasyona atfedilmektedir. Ayrıca Şekil 5 'in içerisinde gösterilen, $420 \mathrm{~nm}$ deki küçük emisyon piki yakın bant kenarı emisyonu (the near-band-edgeNBE) ile ilişkilidir [29].

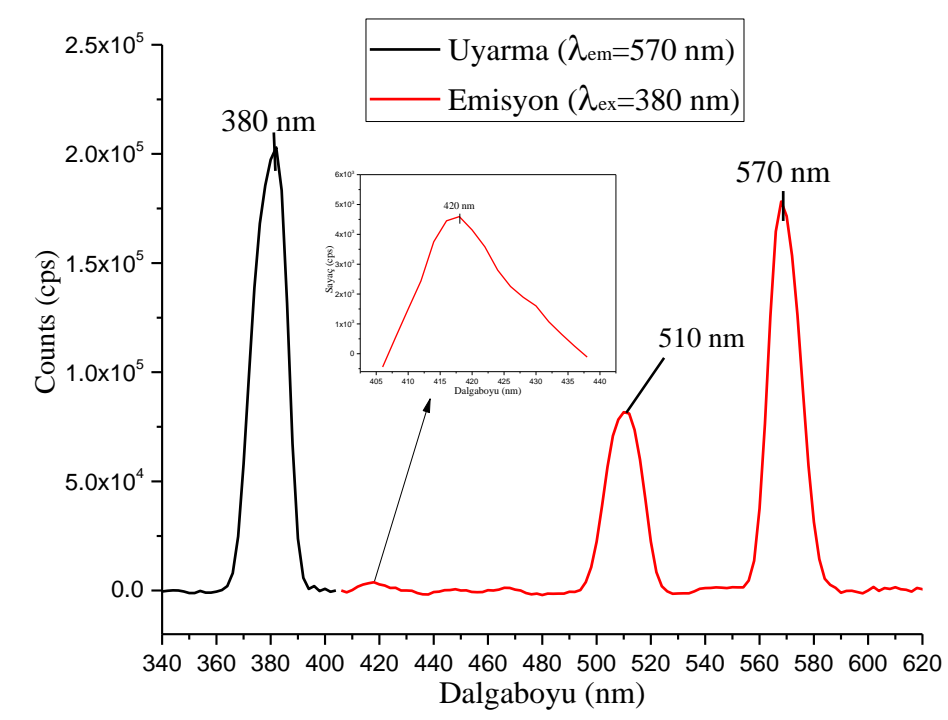

Şekil 5. IGZO_1200 nanopartiküllerinin uyarma ve emisyon bandları

Oda sıcaklığında 1şıldama bozulma süreleri, IGZO nanopartikülleri için kaydedildi (Bkz. Tablo 3 ve Şekil 6). Partiküller, $380 \mathrm{~nm}$ 'de bir mikrosaniye flaş lamba ile uyarıldı ve emisyonlar $570 \mathrm{~nm}$ 'de topland1. Nanopartiküller iki üstel bozulma zamanı gösterdi. 9,99 $\mu$ s kısa ömürlü ve $262 \mu$ s lik orta ömürlü olarak kaydedildi. İlk bozulma sığ tuzaklardan kaynaklanırken, ikinci bozulma ise derin tuzaklar nedeniyle olduğu düşünülmektedir.

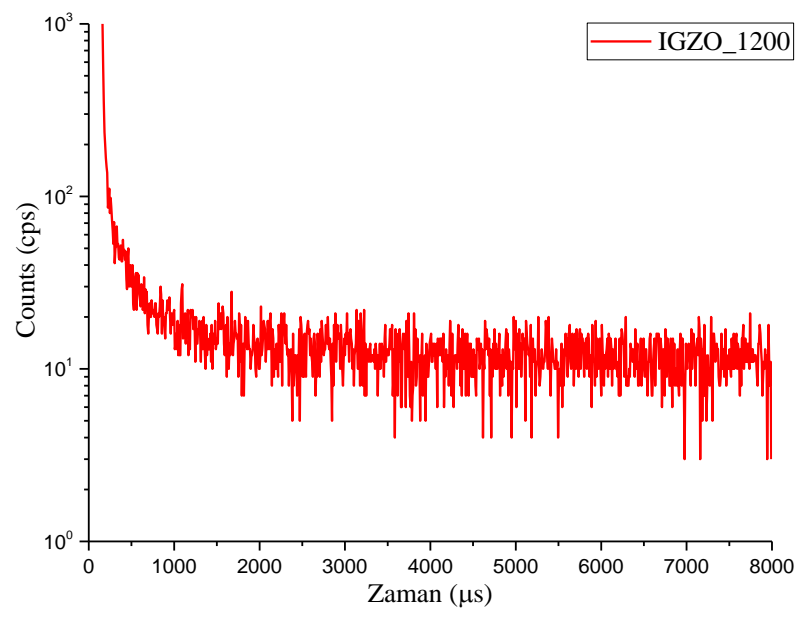

Şekil 6. IGZO_1200 nanopartiküllerinin optik bozulma eğrisi

Tablo 3. Uyarma, emisyon dalga boyu ve standart sapmalar ve yüzde dağılımı ile IGZO partiküllerin optik bozulma zamanı ölçüm sonuçları.

\begin{tabular}{|c|c|c|c|c|c|c|}
\hline Numune Ad 1 & $\lambda_{\max }^{e x}, \lambda_{\max }^{e m}$ & $\chi^{2}$ & & Boz. Zaman $(\mu \mathrm{s})$ & Standat Sap. $(\mu \mathrm{s})$ & Rel. \% \\
\hline \multirow{3}{*}{ IGZO_1200 } & \multirow{3}{*}{$\begin{array}{l}\text { Ex: } 380 \mathrm{~nm} \\
\text { Em:570 nm }\end{array}$} & \multirow{3}{*}{1,392} & $\tau_{1}$ & 9,99 & $\pm 0,07$ & 91,14 \\
\hline & & & $\overline{\tau_{2}}$ & 262,49 & $\pm 9,46$ & 8,86 \\
\hline & & & $\tau_{\text {or }}$ & $32,36 \mu \mathrm{s}$ & & \\
\hline
\end{tabular}




\section{Sonuçlar}

Bu FSP üretimi sonrası başarılı bir şekilde IGZO partikülleri nanopartikül olarak üretilmiştir. Ayrıca elementsel analiz sonucunda yapının kimyasal oranı doğrulanmış ve herhangi bir kirlilik içermediği gözlemlenmiştir. Faz analizi sonrası rombohedral $\mathrm{InGaZnO}_{4}$ yapısı başarılı bir şekilde bulunmuştur. Şeffaf ve esnek ekran teknolojilerinde önemli bir yeri olan IGZO malzemesinin 510 ve $570 \mathrm{~nm}$ yeşil bölgede lüminesans özellik sergilediği görülmüsstür.

\section{Teşekkür}

$\mathrm{Bu}$ çalışmanın yapıldığı ve cihazlarının kullanıldığı Elektronik Malzemeler Üretimi ve Uygulama Merkezi (EMUM)' ne vermiş olduğu desteklerden dolayı teşekkür ederim.

\section{Yazarların Katkısı}

Tüm deneysel çalışmalar ve makale içeriği sorumlu yazar tarafından yapılmıştır.

\section{Araştırma ve Yayın Etiği Beyanı}

Yapılan çalışmada araştırma ve yayın etiğine uyulmuştur.

\section{Kaynaklar}

[1] Zhang L., Zhang H., Bai Y., Ma J.W., Cao J., Jiang X.Y., Zhang Z.L. 2008. Enhanced performances of ZnO-TFT by improving surface properties of channel layer. Solid State Communications, 146 (9-10): 387-390.

[2] Kumaresan Y., Pak Y., Lim N., Lee R., Song H., Kim T.H., Choi B., Jung G.Y. 2016. Effect of channel thickness, annealing temperature and channel length on nanoscale $\mathrm{Ga}_{2} \mathrm{O}_{3}-\mathrm{In}_{2} \mathrm{O}_{3}-\mathrm{ZnO}$ thin film transistor performance. Journal of Nanoscience and Nanotechnology, 16 (6): 63646367.

[3] Sun J., Lu A., Wang L., Hu Y., Wan Q. 2009. High-mobility transparent thin-film transistors with an Sb-doped nanocrystal channel fabricated at room temperature. Nanotechnology, 20 (33): 335204.

[4] Meng Y., Liu G., Liu A., Song H., Hou Y., Shin B., Shan F. 2015. Low-temperature fabrication of high performance indium oxide thin film transistors. RSC Advances, 5 (47): 37807-37813.

[5] Fujii M., Ishikawa Y., Ishihara R., Cingel J. van der, Mofrad M.R.T., Horita M., Uraoka Y. 2013. Low temperature high-mobility InZnO thin-film transistors fabricated by excimer laser annealing. Applied Physics Letters, 102 (12): 122107.

[6] Dai S., Wang T., Li R., Wang Q., Ma Y., Tian L., Su J., Wang Y., Zhou D., Zhang X., Wang Y. 2018. Preparation and electrical properties of N-doped $\mathrm{ZnSnO}$ thin film transistors. Journal of Alloys and Compounds, $745: 256-261$.

[7] Chuang C.-S., Fung T.-C., Mullins B.G., Nomura K., Kamiya T., Shieh H.-P.D., Hosono H., Kanicki J. 2008. P-13: Photosensitivity of amorphous IGZO TFTs for active-matrix flat-panel displays. SID Symposium Digest of Technical Papers, 39 (1): 1215-1218.

[8] Hsieh H., Lu H., Ting H., Chuang C., Chen C., Lin Y. 2011. Development of IGZO TFTs and their applications to next-generation flat-panel displays. Journal of Information Display, 11 (4): 160-164.

[9] Jung C., Choi M.S., Choi K.H., Yoon D.H. 2010. Controllable crystallinity of synthesized InGa-Zn-O nano-powder by using a pulp precursor for printing processes. Physica Status Solidi (A), 207 (7): 1680-1683.

[10] Wu M.C., Hsiao K.C., Lu H.C. 2015. Synthesis of $\mathrm{InGaZnO}_{4}$ nanoparticles using low temperature multistep co-precipitation method. Materials Chemistry and Physics, 162 :386-391.

[11] Fukuda N., Watanabe Y., Uemura S., Yoshida Y., Nakamura T., Ushijima H. 2014. In-Ga-Zn oxide nanoparticles acting as an oxide semiconductor material synthesized via a coprecipitationbased method. Journal of Materials Chemistry C, 2 (13): 2448-2454.

[12] Barquinha P., Pereira L., Gonçalves G., Martins R., Fortunato E. 2009. Toward high- 
performance amorphous GIZO TFTs. Journal of The Electrochemical Society, 156 (3): H161.

[13] Nomura K., Takagi A., Kamiya T., Ohta H., Hirano M., Hosono H. 2006. Amorphous oxide semiconductors for high-performance flexible thin-Film transistors. Japanese Journal of Applied Physics, Part 1: Regular Papers and Short Notes and Review Papers, 45 (5 B): 4303-4308.

[14] Jeong J.K., Jeong J.H., Yang H.W., Park J.S., Mo Y.G., Kim H.D. 2007. High performance thin film transistors with cosputtered amorphous indium gallium zinc oxide channel. Applied Physics Letters, 91 (11): 113505.

[15] Park J.S., Jeong J.K., Mo Y.G., Kim H.D., Kim S. Il. 2007. Improvements in the device characteristics of amorphous indium gallium zinc oxide thin-film transistors by ar plasma treatment. Applied Physics Letters, 90 (26): 262106.

[16] Yabuta H., Sano M., Abe K., Aiba T., Den T., Kumomi H., Nomura K., Kamiya T., Hosono H. 2006. High-mobility thin-film transistor with amorphous $\mathrm{InGaZnO}_{4}$ channel fabricated by room temperature Rf-Magnetron sputtering. Applied Physics Letters, 89 (11): 112123.

[17] Iwasaki T., Itagaki N., Den T., Kumomi H., Nomura K., Kamiya T., Hosono H. 2007. Combinatorial approach to thin-film transistors using multicomponent semiconductor channels: an application to amorphous oxide semiconductors in In-Ga-Zn-O system. Applied Physics Letters, 90 (24): 242114.

[18] Nomura K., Ohta H., Takagi A., Kamiya T., Hirano M., Hosono H. 2004. Room-temperature fabrication of transparent flexible thin-film transistors using amorphous oxide semiconductors. Nature, 432 (7016): 488-492.

[19] Cerdà J., Arbiol J., Diaz R., Dezanneau G., Morante J.R. 2002. Synthesis of perovskite-type $\mathrm{BaSnO}_{3}$ particles obtained by a new simple wet chemical route based on a sol-gel process. Materials Letters, 56 (3): 131-136.

[20] Habeeba K., Manjulavalli T.E., Ezhilarasi Gnanakumari D. V., Karthikadevi V. 2019. Highly crystalline perovskite $\mathrm{BaSnO}_{3}$ nanopowder synthesised using hydrothermal technique. Materials Research Express, 6 (9): 094004.

[21] Deepa A.S., Vidya S., Manu P.C., Solomon S., John A., Thomas J.K. 2011. Structural and optical characterization of $\mathrm{BaSnO}_{3}$ nanopowder synthesized through a novel combustion technique. Journal of Alloys and Compounds, 509 (5): 1830-1835.

[22] Gültekin S., Yıldırım S., Yılmaz O., Keskin İ.Ç., Katı M.İ., Çelik E. 2019. Structural and optical properties of $\mathrm{SrAl}_{2} \mathrm{O}_{4}: \mathrm{Eu}^{2+} / \mathrm{Dy}^{3+}$ phosphors synthesized by flame spray pyrolysis technique. Journal of Luminescence, $206: 59-69$.

[23] Yildirim S., Yurddaskal M., Dikici T., Aritman I., Ertekin K., Celik E. 2016. Structural and luminescence properties of undoped, $\mathrm{Nd}^{3+}$ and $\mathrm{Er}^{3+}$ doped $\mathrm{TiO}_{2}$ nanoparticles synthesized by flame spray pyrolysis method. Ceramics International, 42 (9): 10579-10586.

[24] Yildirim S., Karsu Asal, E.C., Ertekin K., Celik E. 2017. Luminescent properties of scintillator nanophosphors produced by flame spray pyrolysis. Journal of Luminescence, 187 : 304-312.

[25] Liu J.A., Li C.H., Shan J.J., Wu J.M., Gui R.F., Shi Y.S. 2018. Preparation of high-density $\mathrm{InGaZnO}_{4}$ target by the assistance of cold sintering. Materials Science in Semiconductor Processing, $84: 17-23$.

[26] Chen J., Wang L., Su X., Kong L., Liu G., Zhang X. 2010. InGaZnO semiconductor thin film fabricated using pulsed laser deposition. Optics Express, 18 (2): 1398.

[27] Krishnan R., Thirumalai J., Chandramohan R. 2013. Room temperature photo-induced, Eu ${ }^{3+}$ doped IGZO transparent thin films fabricated using sol-gel method. Journal of Nanostructure in Chemistry, 3 (1): $1-4$.

[28] Wu G.M., Liu C.Y., Sahoo A.K. 2015. RF sputtering deposited A-IGZO films for LCD alignment layer application. In: Applied Surface Science. Elsevier B.V., pp 48-54.

[29] Xu W., Hu L., Zhao C., Zhang L., Zhu D., Cao P., Liu W., Han S., Liu X., Jia F., Zeng Y., Lu Y. 2018. Low temperature solution-processed IGZO thin-film transistors. Applied Surface Science, 455: 554-560. 